\title{
Protein-protein interactions: better by the dozen
}

A combined reanalysis of the two largest yeast protein-protein interaction studies to date provides a large consolidated data set, with a level of accuracy matching the reliability of small-scale experiments.

Last year, the groups of Giulio SupertiFurga and Jack Greenblatt published two yeast interactome studies by systematic tandem affinity purification (TAP) of epitopetagged proteins followed by mass spectrometry analysis (Gavin et al., 2006; Krogan et al., 2006). Nevan Krogan, who was the lead author of one of the studies, has now merged the two data sets and reanalyzed them using Bayesian statistics (Collins et al., 2007).

This novel analysis, which was done collaboratively with Sean Collins and Jonathan Weissman, assigns a probability-based score to each interaction taking into account repetitions of observations in the two independent studies. For a protein pair A-B, observed when $\mathrm{A}$ is epitope-tagged, the score is higher if $\mathrm{B}$ is observed in multiple replicate purifications of $A$, or if both $A$ and $B$ copurify with a third protein C. Conversely, A-B's score is lower if A does not copurify with epitope-tagged B.

Using two reference sets of well-established interactions, Krogan and colleagues compared the accuracy of the individual and consolidated TAP data sets with a set of interactions extracted from a database of small-scale experiments. As expected, the consolidated data set proved superior to both individual TAP data sets. Most importantly, the $\sim 9,000$ highest-scoring interactions in the consolidated TAP data set demonstrated a level of confidence at least as good as that from small-scale experiments. They validated this 9,000-interaction subset by cross-referencing it to previously published subcellular colocalization data, Gene Ontology annotation and coexpression data from geneexpression profiling. In all cases, the consolidated TAP data set performed at least as well as the small-scale data set.
As Superti-Furga puts it, "it speaks for the necessity of not having only one group do such type of approaches because there is always something to be gained about having this repetition of data." Krogan hopes that this consolidated dataset will help convince biologists of the value of large scale approaches. "The challenge," he says, "is persuading scientists to examine [these] data and convincing them that large-scale data sets can be of high quality. People need to see the physiologically relevant interactions for the proteins they have worked on for 20 years."

\section{Veronique Kiermer}

\section{RESEARCH PAPERS}

Collins, S.R. et al. Toward a comprehensive atlas of the physical interactome of Saccharomyces cerevisiae. Mol. Cell. Proteomics 6, 439-450 (2007).

Gavin, A.C. et al. Proteome survey reveals modularity of the yeast cell machinery. Nature 440, 631-636 (2006).

Krogan, N.J. et al. Global landscape of protein complexes in the yeast Saccharomyces cerevisiae. Nature 440, 637-643 (2006). 